\title{
Flat Panel Ultrovilet Lamps by vehicle of Porous Alumina and ITO glass
}

\author{
Jian $\mathrm{LI}^{1,2, a}$, Kun WANG ${ }^{1,2, b}$, Shi-qing WANG ${ }^{1,2, c}$ \\ ${ }^{1}$ Southwestern Institute of Physics, Chengdu, 610041, China \\ ${ }^{2}$ Engineering and Technical College of Chengdu University of Technology, Leshan, 614007, China \\ a lijwk@126.com, ${ }^{\mathrm{b}}$ wangkun6236@sina.com, ${ }^{\mathrm{c}}$ wsq6222@126.com
}

Keywords: ultroviolet lamps, vacuum violet radition, electron temperature

\begin{abstract}
Lamps with about 20W/m2 UV emission were made in our work. The lamp was fabricated by ITO glass and bonded aluminum with porous alumina on the surface. The working gas is the mixture of argon and Nitrogen. Lamps properties were investigated.
\end{abstract}

\section{Introduction}

Dielectric barrier discharge (DBD), also referred to as barrier discharges or silent discharges have found a number of interesting industrial applications in addition to the historical ozone generation. Dielectric barrier discharge is important in that it can easily produce non-thermal plasma in an easy way. Most of all, it has been used widely in the PDP production. Argon and Nitrogen mixture is good for ultraviolet emission. Ultraviolet (UV) and Vacuum Ultraviolet (VUV) radiation are important applications [1-5]. Usually the UV lamps are tube sources. The flat panel UV source are seldom designed and referred[6, 7].

In our work, a flat panel UV lamp was fabricated by using ITO glass as the transparent electrode and dielectric, bonded aluminum with porous alumina acts as the other electrode. The properties of the discharge are investigated. With nitrogen concentration increasing at 0.5-20 percent, the UV emission decreases at the same voltage. However, the UV emission properties in the total emission dropped meanwhile. The spectra of the lamp were also taken and analysized.

\section{Experiment}

Figure 1 shows the flat panel UV lamp. The 0.56-mm-thick ITO glass acts as the front electrode and the dielectric of the UV lamp. The rear electrode is made of bonded aluminum with porous alumina on the surface. The distance between the front and rear electrode is $0.44 \mathrm{~mm}$. The aluminum thickness is $50 \mathrm{~m}$, and the alumina, which is produced by anodizing in the oxalic acid, is $5 \mathrm{~m}$. After anodizing, the porous AA is eroded by voltage drop till the voltage is $3 \mathrm{~V}$. Pore widening in $0.1 \mathrm{M}$ H3PO4 solution is carried out afterwards. Nickel pigmented the porous alumina in the end to increase the alumina thickness.

The UV lamp was filled up with Ar-N2 mixture after the lamp was vacuumed till 10-7torr. In discharge, the gas mixtures were excited in a pulsed transverse power supply. The power supply repetition is $20-40 \mathrm{kHz}$ and the peak voltage is about $15 \mathrm{kv}$. The spectra were taken in the course of the discharge.

The power density was tested by a power meter with and without the SRG-610 filter. The Power Meter is 2832-C Dual-Channel Power Meter (produced by Newport Company). SRG-610 filter can separate the emission into two parts: 200-610 nm and above $610 \mathrm{~nm}$. So we can test the total emission power density without SRG-610 filter and the near IR emission (above $610 \mathrm{~nm}$ ) power density with the filter. Therefore, the UV emission power density can be attained by subtracting the near IR emission power density from total one. 

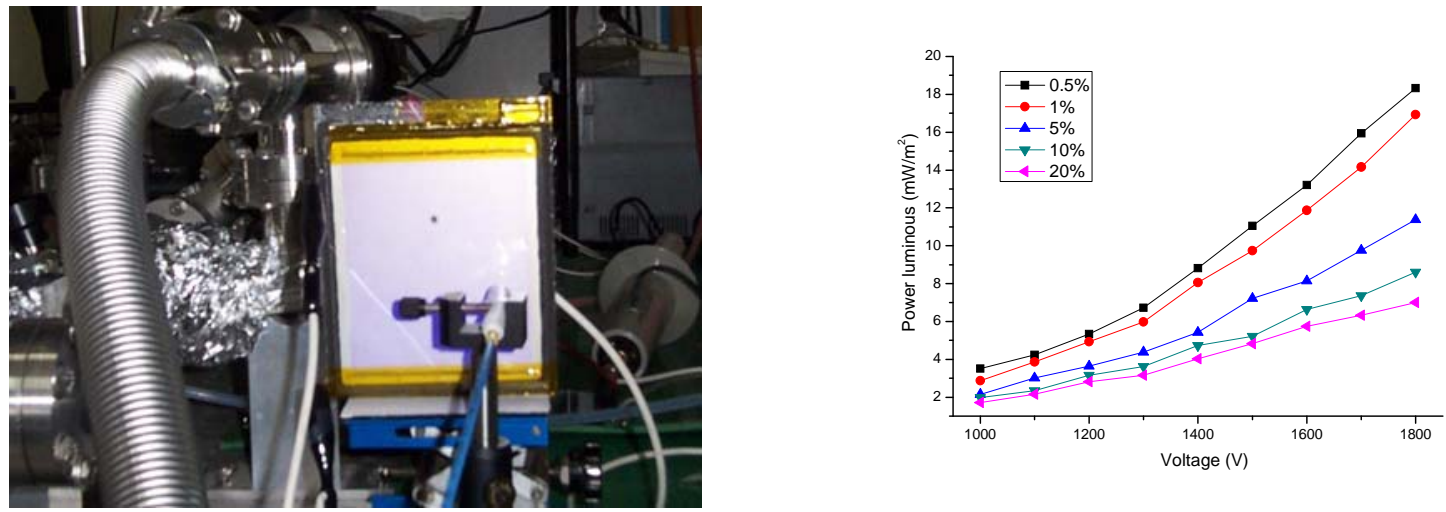

Figure 1. Flat panel UV lamps

Fig.2. Power density versus voltage at different $\mathrm{N}_{2}$ concentrations

\section{Result and discussion}

In Ar-N2 mixture discharge, when N2 is of small quantity, the excited species are mainly atomic argon ions ( $\mathrm{Ar}+$ ), electrons (e-), and the excited argon species in radiative or metastable states. It was believed that the Ar metastables (Arm) is the common energy source for all the species emitting ultraviolet (UV) and vacuum ultraviolet (VUV). Nitrogen can get energy, and turn it into active species and give out UV emission [8].

$\mathrm{Ar}$ alone can only give out near IR emission in the lamps. Because of the lower concentration, Nitrogen cannot have an appreciable influence on the argon metastable states processes populating. However, it can efficiently depopulate these states in the reaction:

$$
A r^{m}+N_{2} \rightarrow N_{2}^{*}+A r
$$

where N2* represent all possible molecular excited states, which can give out UV emission.

The UV Power and Nitrogen concentration.Figure 2 shows the curve of the UV power density vs. voltage at different Nitrogen concentration. The testing condition is: 0.5 to 20 per cent Nitrogen in the mixture, total pressure 200 torr, the power supply repetition $40 \mathrm{kHz}$. With the Nitrogen concentration increasing, at the same voltage, the UV emission will decrease and the UV emission proportion in the total emission will increase.

In the working gas, the Arm concentration drops with Nitrogen concentration increasing[9-12]. As the Nitrogen is lower, the quantity of argon metastable states is enough to excite the Nitrogen molecules to give out UV. So Arm can excite the Nitrogen molecules more effectively. More UV emission will be given out.
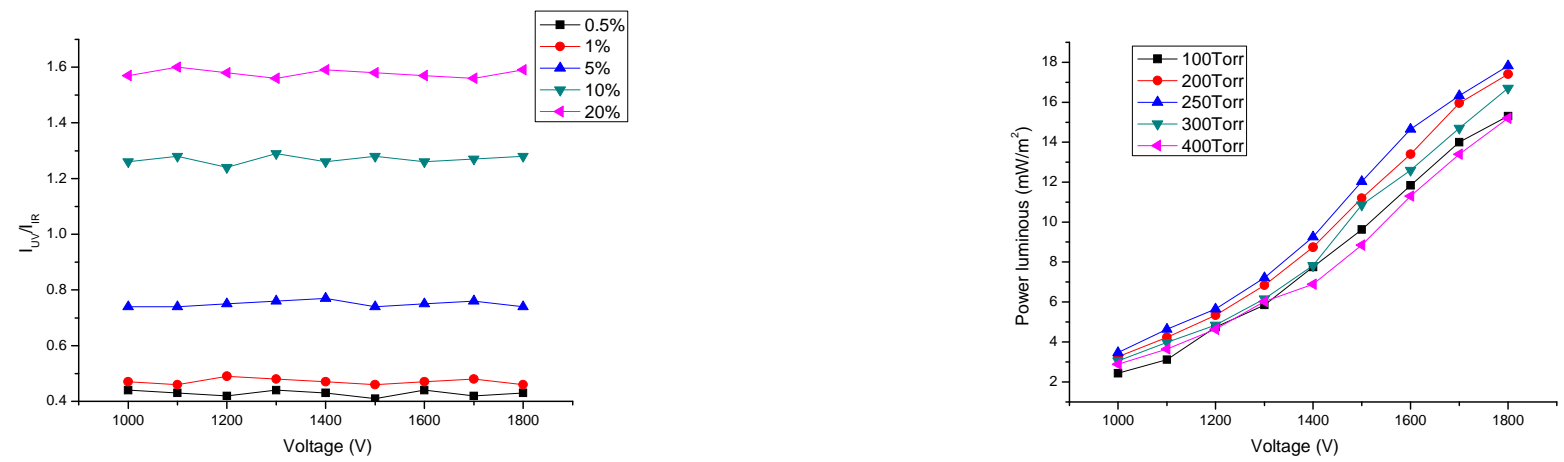

Fig3. UV concentration in radiation versus voltage at different $\mathrm{N}_{2}$ concentrations Figure 5. Power density versus voltage at different $\mathrm{N}_{2}$ concentrations

Meanwhile, lower Nitrogen concentration will result in more argon relative species present, which give out higher near IR emission. The UV emission power in the total emission dropped with Nitrogen concentration increasing. This can be proven in the lamp spectra, which is shown in Figure 3.

Figure 4 shows the spectra of the UV lamp. There are mainly two parts of spectra lines which locates at UV region and near IR region. They are Nitrogen related species and Argon related species 
respectively. The near IR emission power density at 1 percent Nitrogen is more intense than that at 5 percent. When a pulsed discharge of a short duration excites the mixture of Argon and a small amount of Nitrogen, the excited species in the plasma are mainly Argon related species. Argon metastables (Arm), which has longer lifetime, act as the energy source of the UV and VUV emission [13-15]. In our experiment, the UV emission acceptors are mainly Nitrogen molecules. Arm also provides energy to the other Argon related species and gives out near IR emission. So a competition occurs between Ar and N2 species. When N2 concentration increases, more N2 molecules existing in the mixture will cause higher UV emission power proportion. However, as Ar concentration dropping, Arm concentration drops. The total UV power density decreases too.
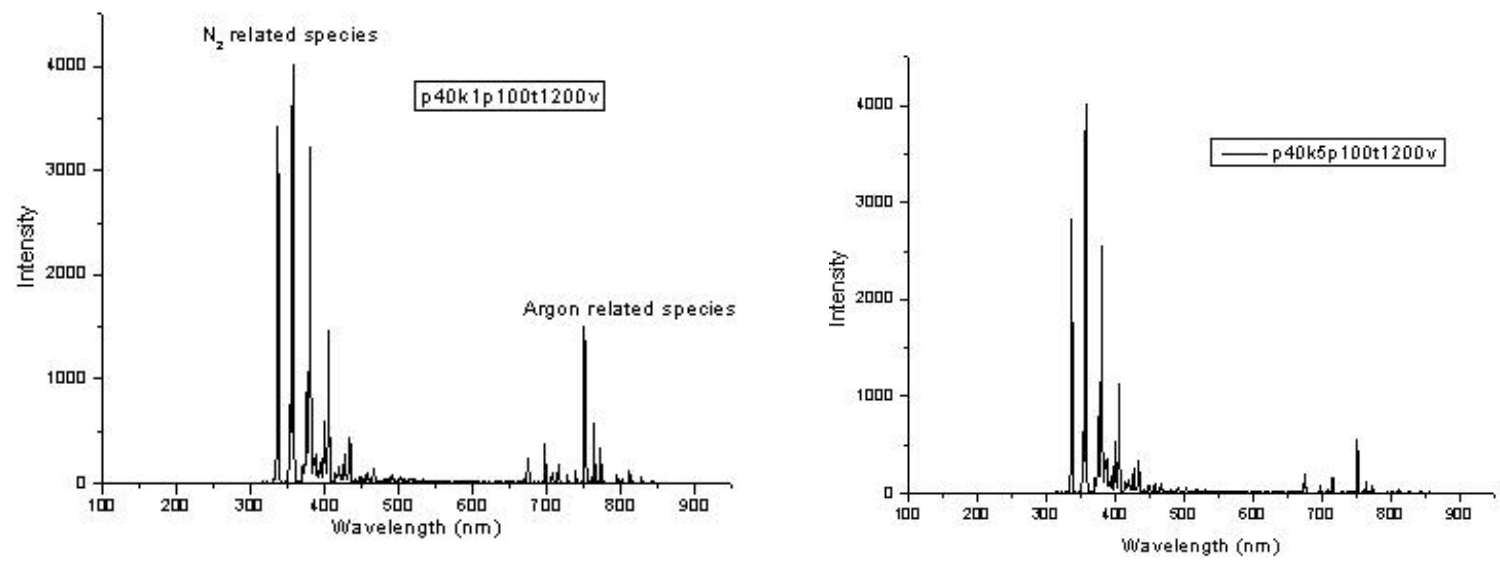

Figure 4. The UV lamp spectra: 1 and 5 percent nitrogen in the Ar- $\mathrm{N}_{2}: 100$ torr; $1200 \mathrm{v}, 40 \mathrm{kHz}$.

The UV power and pressure.Figure 5 shows the experimental curves of UV power density and voltage at different pressures. The pulsed power supply repetition is $40 \mathrm{kHz}$. The UV lamp performs better when the pressure is at 200 and 300 torr. From 200 to 400 torr, the UV power density emission performance becomes worse, which is similar to that of 100torr.

In Ar-N2 discharge, the UV emission performance rests on the quantities excited N2. Excited Nitrogen molecules get energy from Arm by collisions. Therefore, Nitrogen molecules and Arm concentration is the key to UV emission performance [16]. At 100 torr or lower pressure, there are not enough Nitrogen molecules colliding with Arm, so that more argon related species are excited and give out more near IR emission. At 200-300 torr, the Nitrogen molecules are just suitable, and the lamp will give out more UV emission and less near IR emission than at 100 torr[17].

From the spectra100 and 300 torr, more IR emission produces produced at 100torr than that of 300torr. Therefore, we can conclude the N2 molecules absolute concentration at 300 torr is more suitable for the collisions between N2 molecules and Arm than that at 100 torr. More UV emission is produced at 300 torr.

If the pressure goes on increasing, the plasma properties will change: the electron free path will shorten and the number of the excited species will reduce. So the lamp will perform worse when the pressure gets to 400 torr or above.

\section{Conclusion}

Flat panel UV lamps which can give out above $20 \mathrm{~mW} / \mathrm{m} 2 \mathrm{UV}$ emission are made in our laboratory by using ITO glass and bonded aluminum with porous alumina as the former and rear electrode structures. The lamps can emit intense UV emission by vehicle of Ar-N2 discharge. At 200-300 torr and less than 1 percent N2 in Ar-N2 mixture, the lamps UV emission performance is much better than those at the other conditions.

\section{References}

[1] R. P. Midren, R. J. Carman. Enhanced performance of a dielectric barrier discharge lamp using short-pulsed excitation, J. Phys. D: Appl. Phys. 34, pp.L1-L6, 2001. 
[2] Wang Zhan, Ren Chunsheng, Nie Qiuyue, et al. Effects of airflows on dielectric barrier discharge in air at atmospheric pressure, Plasma Science and Technology, vol. 11, no. 2, pp. 177-180, 2009.

[3] Zhou, T. M., X. Zhou, and W. X. Cai, Principle and Design of Light Sources, Fudan University Press, Shanghai, China, 2008.

[4] A. Heikkilä, P. Kärhä, A. Tanskanen, M. Kaunismaa, Characterizing a UV chamber with mercury lamps for assessment of comparability to natural UV conditions, Polymer Testing,Vol. 28, no. 1, pp.57-65,2009.

[5] M. Anandan, D. Ketchum,Present status and future trends of multichannel hollow cathode true flat fluorescent lamp for full-colour LCD backlighting, Displays, Vol. 15, no. 1, January, pp.17-26, 1994.

[6] A. Klausch, H. Althues, T. Freudenberg, S. Kaskel Wet chemical preparation of YVO4:Eu thin films as red-emitting phosphor layers for fully transparent flat dielectric discharge lamp ,Thin Solid Films, Volume 520, Issue 13, pp.4297-4301, 2012.

[7] Q. Fang, G. He, W.P. Cai, J.-Y. Zhang, Ian W. Boyd, Palladium nanoparticles on silicon by photo-reduction using $172 \mathrm{~nm}$ excimer UV lamps Original Research Article, Applied Surface Science, vol. 226, no. 1-3, pp.7-11, 2004.

[8] Anne P. Thorne Calibration of line width standards and lamp intensities using FT UV Original Research Article, Analytical Spectroscopy Library, vol. 6, pp.355-367, 1995.

[9] Ian W. Boyd, Jun-Ying Zhang, Photo-induced growth of dielectrics with excimer lamps Original Research Article, Solid-State Electronics, vol. 45, no. 8, pp. 1413-1431, 2001.

[10] Jun-Ying Zhang, Boon Lim, Ian W Boyd, Deposition and annealing of tantalum pentoxide films using 172 nm excimer lamp Original Research Article, Applied Surface Science, vol. 154-155, pp.382-386, 2000.

[11] Shanshan Jin, Karl G. Linden, Joel Ducoste, Dong Liu, Impact of lamp shadowing and reflection on the fluence rate distribution in a multiple low-pressure UV lamp array Original Research Article, Water Research, vol. 39, no. 12, pp.2711-2721, 2005.

[12] Wei Zhang, Chang-Ying Xue, Kun-Lin Yang ,A method of printing uniform protein lines by using flat PDMS stamps Original Research Article, Journal of Colloid and Interface Science, vol. 353, no. 1, pp.143-148, 2011.

[13] Isaac W. Wait, Cliff T. Johnston, Ernest R. Blatchley III, The influence of oxidation reduction potential and water treatment processes on quartz lamp sleeve fouling in ultraviolet disinfection reactors Original Research Article, Water Research, vol. 41, no. 11, pp. 2427-2436, 2007.

[14] J.J. Yu, I.W. Boyd, UV detection for excimer lamps using CVD diamond in various gaseous atmospheres Original Research Article,Diamond and Related Materials, vol. 16, no. 3, pp.494-497, 2007.

[15] R. Portela, S. Suárez, R.F. Tessinari, M.D. Hernández-Alonso, M.C. Canela, B. Sánchez, Solar/lamp-irradiated tubular photoreactor for air treatment with transparent supported photocatalysts Original Research Article, Applied Catalysis B: Environmental, vol. 105, no. 1-2, pp.95-102, 2011.

[16] Kenji Toda, Recent research and development of VUV phosphors for a mercury-free lamp Original Research Article, Journal of Alloys and Compounds, vol. 408-412, Pages 665-668, 2006.

[17] J. Esteban Duran, Fariborz Taghipour, Madjid Mohseni, Journal of Photochemistry and Photobiology A: Chemistry, vol. 215, no. 1, pp.81-89, 2010. 